\title{
The prevalence of hypoglycemia and its impact on the quality of life (QoL) of type 2 diabetes mellitus patients (The HYPO Study)
}

\author{
Grigorios Rombopoulos, ${ }^{1}$ Magdalini Hatzikou, ${ }^{1}$ Dimitra Latsou, ${ }^{2}$ John Yfantopoulos ${ }^{2}$
}

${ }^{1}$ Medical Dept. \& Health Economics, Novartis Hellas SA, Athens, ${ }^{2}$ University of Athens, Political Science \& Public Administration, Greece

\begin{abstract}
OBJECTIVE: To estimate the impact of hypoglycemia on the Quality of Life (QoL) and its prevalence in patients with type 2 diabetes mellitus (T2DM) in Greece. DESIGN: A crosssectional epidemiological study was conducted in 6631 patients with T2DM. QoL was assessed with the patient self-administered ADDQoL-19. Reliability analysis of patients' outcomes was performed to assess internal consistency of the ADDQoL-19. Patients were categorized according to hypoglycemia experience and diabetes control. Controlled patients were considered as having Hb1Ac $<7 \%(53 \mathrm{mmol} / \mathrm{mol})$ and hypoglycemic episodes were defined as laboratoryconfirmed clinical symptomatic events. RESULTS: In total, 59\% of the sample had HbA1c $>7 \%$ $(53 \mathrm{mmol} / \mathrm{mol})$ and $20.4 \%$ of patients had a history of laboratory-confirmed hypoglycemia. The mean age was 60 years and the mean T2DM duration was 10 years. The mean QoL score was $-3.09 \pm 1.9$ for the total sample and the mean score of non-hypoglycemic patients was $-3.05 \pm 2$, while the respective score of hypoglycemic patients was $-3.26 \pm 1.8,(p \leq 0.001)$. Similar results were observed in the group of controlled and uncontrolled patients, who scored $-2.73 \pm 1.7$ and $-3.33 \pm 1.9$, respectively $(p \leq 0.001$ ). Comparable results were identified in the majority of the ADDQoL-19 instrument dimensions. CONCLUSIONS: The QoL of T2DM patients is affected significantly by hypoglycemia and the level of disease control. In diabetes treatment, the ultimate goal should be optimal glycemic control without debilitating hypoglycemic episodes which compromise patients' QoL.
\end{abstract}

Key words: ADDQoL-19, Greece, Hypoglycemia, Quality of Life, Type 2 Diabetes

Address for correspondence:

Grigorios Rombopoulos MD, $12^{\text {th }} \mathrm{klm}$ National Road 1,

Metamorfosis, 14451, Athens, Greece,

Tel.: +30 2102897204, Fax: +302102897310,

e-mail: grigorios.rombopoulos@novartis.com

Received 31-07-2013, Accepted 15-10-2013

\section{INTRODUCTION}

Type 2 diabetes mellitus is a chronic progressive disease characterized by hyperglycemia resulting from multiple abnormalities, including impaired insulin secretion, increased hepatic glucose production, 
hyperglucagonemia and peripheral insulin resistance. Diabetes is a major public health concern, as it leads to serious long-term micro- and macrovascular complications that increase morbidity, mortality and healthcare costs. ${ }^{1}$

According to the International Diabetes Federation, diabetes mellitus is becoming a globally epidemic disease with more than 366 million patients affected, while this number is expected to reach 552 million by 2030. This prediction indicates a significant increase both in the percentage of people with diabetes as well as in the prevalence of the disease. ${ }^{1}$ In 2012, Greece was estimated as having about 638 thousand diabetic patients and a prevalence rate of $5.22 \%$, based on the IDF atlas. ${ }^{1}$ Higher rates were reported in an epidemiological study performed in Greece in 2002 where the estimated prevalence of T2DM was $7.6 \%$ in men and $5.9 \%$ in women. ${ }^{2}$ A significant proportion of diabetic subjects $(24 \%$ of men and $30 \%$ of women) were unaware of their condition, while a 10-unit increase in the diet score was associated with $21 \%$ lower odds of diabetes $(\mathrm{P}<0.05){ }^{2}$

Hypoglycemia is a common, unpredictable and potentially dangerous side effect of diabetes therapy. The greatest clinical risk results from a lack of adequate cerebral glucose supply causing progressive cognitive impairment, confusion and finally coma. ${ }^{3}$ Hypoglycemia is also associated with lower QoL in T2DM patients. In particular, this can be seen through the events caused by this adverse reaction, the fear of this, as well as through fear of possible long-term effects. ${ }^{4}$ Therefore, it is evident that both diabetes and hypoglycemia negatively affect the patients' wellbeing as well as their QoL. ${ }^{4-7}$

QoL is a multidimensional phenomenon. However, there is no concrete conceptual approach incorporating all the possible social, economic, psychological and health dimensions into a single indicator. In the literature, different instruments for assessing the general health of a population (generic instruments) as well as the clinical implications of several treatment therapies (disease specific instruments) have been used. T2DM can be objectively measured through clinical assessment of patients' health status, but also by taking into account the patients' subjective views on various dimensions which affect their QoL. Specifically designed questionnaires are used for the measurement of subjective evaluation of health. ${ }^{8}$ It is worth mentioning that the impact assessment of T2DM on QoL and well-being of patients has been the subject of research in both clinical and health economic research. Furthermore, patients with diabetes have impaired physical and mental well-being, while they also feel emotional distress and helplessness. ${ }^{9-13}$ It is noteworthy that patients with diabetes have lower levels of QoL than their healthy peers, ${ }^{14-16}$ while the progress of disease and its complications negatively affect the improvement of QoL. ${ }^{17,18}$ For assessment of health status, the above items are added to demographic characteristics, obesity, hypertension and any pre-existing heart problems. ${ }^{19}$ The relation between T2DM and socioeconomic status and its impact on the lower income classes has been recently investigated by clinicians and health economists. ${ }^{20,21}$ Cost effectiveness analysis has been used to evaluate the impact on an individual's budget and the expenses involved for the improvement of QoL. ${ }^{22}$

The objective of this study was to estimate the prevalence of hypoglycemia in type 2 diabetic patients and its impact on their quality of life.

\section{MATERIALS AND METHOD}

The present study was a cross-sectional epidemiological research conducted in 6631 Greek patients with T2DM within a 6-month time period. The study population was selected by a random sampling in accordance with geographical distribution around Greece.

The ADDQoL-19 QoL instrument was self-administered and completed by each patient. Patient demographic data, medical history, number of hypoglycemic episodes and historical measurements of $\mathrm{Hb} 1 \mathrm{Ac}$ were analyzed by internists and endocrinologists specialized in diabetes. Patients' categorization was based on the following parameters: experience of hypoglycemia or not and controlled or uncontrolled. Controlled patients were considered as having $\mathrm{Hb} 1 \mathrm{Ac}<7 \%$ $(53 \mathrm{mmol} / \mathrm{mol})$. Hypoglycemic episodes were defined as laboratory-confirmed clinical symptomatic events.

Each patient completed one questionnaire concerning his/her QoL [Greek edition of the Audit 
of Diabetes Dependent QoL Questionnaire (ADDQoL-19)]. Additionally, each physician filled in the patients' clinical characteristics and glycated hemoglobin (HbA1c) as well as providing a questionnaire related to hypoglycemia symptoms, severity and frequency during the previous year.

The ADDQoL-19 instrument was designed and developed by Bradley et al., 1999, in order to measure the individual's perception of HRQL when suffering from diabetes. The questionnaire assesses 19 domains: leisure activities, working life, travel, holiday, physical activities, family life, social life, personal life, sex life, physical appearance, self-confidence, motivation, reaction from others, feelings about the future, financial situation, living conditions, reliance on others, freedom to eat and freedom to drink. The score of each item ranged from -9 to 3 with a lower score indicating a worse health state. Finally, the questionnaire includes two overview items which report the general QoL. ${ }^{23}$

In addition, the data were analyzed using the statistical program SPSS 17.00. The demographic characteristics (gender, age, duration of disease, Body Mass Index, $\mathrm{HbA1c}$ ) of the sample were presented as frequencies and rates. The mean value and standard deviation were used for the quality-of-life parameters.

The assessment of the psychometric properties of the HRQL instrument, and in particular reliability, was performed using Cronbach's coefficient $a_{\text {Cronbach }}$.

Cronbach's $a$ has been proven to underestimate the true reliability and is therefore a conservative measurement. Thus, the 0.7 alpha coefficient is considered as a cut-off value, above which the HRQL questionnaire is considered reliable. ${ }^{24}$

The statistical significance of the results was investigated using the chi-square $\left(\mathrm{x}^{2}\right)$ test (for percentages) and the mean values were compared using the Student's t-test when the variables followed the normal distribution, and the Mann-Whitney U-test when the variables were not normally distributed.

Finally, the correlation analysis was conducted using the Pearson $R$ coefficient for normally distributed variables, and the Spearman coefficient for non-normally distributed variables. The instructions of Cohen were used for the interpretation of these coefficients. Specifically, it was assumed that a coef- ficient of 0.10 indicates low correlation, a coefficient of 0.30 indicates moderate correlation and a coefficient of 0.50 reveals a high correlation. ${ }^{25}$

\section{RESULTS}

In total, 6631 patients with T2DM were enrolled in the study. The age groups ranged from 19 to more than 90 years old. $35.1 \%$ of the patients were $61-70$ and $30 \%$ were in the age group of 51-60. In total, 2979 patients $(45 \%)$ were female, while 3642 patients $(55 \%)$ were male. As far as Body Mass Index is concerned, $59 \%$ (3890) of the patients were overweight, followed by $22.5 \%$ (1481) who were characterized as obese class I (BMI 30-35). $44.4 \%$ of patients had suffered from the disease from 0 to 5 years and $27.8 \% 6$ to 10 years. According to the last $\mathrm{HbA} 1 \mathrm{c}$ test results, $41 \%$ of patients were controlled, while $59 \%$ were uncontrolled. Specifically, 2957 (44.7\%) patients were moderately uncontrolled (7-7.99\%/53-64 mmol/ $\mathrm{mol})$ and the remaining $943(14.3 \%)$ were severely uncontrolled ( $>8 \% / 64 \mathrm{mmol} / \mathrm{mol}$ ) (Table 1).

$41.9 \%$ of patients $(n=2779)$ stated that they had experienced the symptoms of hypoglycemia during the last three months, whereas only $20.4 \%(n=1.354)$ had laboratory-confirmed hypoglycemia. The disposition of $41.9 \%$ is analyzed as follows: $25.5 \%$ with only one hypoglycemic event, $11.1 \%$ with two, while lower percentages were observed in patients with more than three events during the last three months. Finally, $11.6 \%$ (767) of patients had experienced severe hypoglycaemia and were transferred to hospital (Table 2).

The disposition of hypoglycemic events among the controlled and uncontrolled patients is presented in Table 3. Between the hypoglycemic and the non-hypoglycemic population there was a statistical significant difference $(p=0.001)$ in HbA1c levels. The group of uncontrolled patients had 1.419-fold increased risk of having hypoglycemic events, in comparison to the controlled patients (CI: 1.610-1.971).

The impact of diabetes on QoL of the patients of the whole study sample, i.e. the hypoglycemic and non-hypoglycemic groups as well as the controlled and uncontrolled groups, is presented in figure 1. Lower QoL scoring was observed in hypoglycemic and uncontrolled patients. A statistically significant difference was found between hypoglycemic and non-hypoglycemic patients, as well as between the 
Table 1. Demographic and disease characteristics

\begin{tabular}{|c|c|c|c|}
\hline & & Number & Percentage \\
\hline \multirow[t]{8}{*}{ Age group (years) } & $19-30$ & 18 & 0.3 \\
\hline & $31-40$ & 154 & 2.3 \\
\hline & $41-50$ & 844 & 12.7 \\
\hline & $51-60$ & 1984 & 30.0 \\
\hline & $61-70$ & 2326 & 35.1 \\
\hline & $71-80$ & 1130 & 17.1 \\
\hline & $81-90$ & 157 & 2.4 \\
\hline & $90+$ & 8 & 0.1 \\
\hline \multirow[t]{2}{*}{ Gender } & Male & 3642 & 55 \\
\hline & Female & 2979 & 45 \\
\hline Body Mass & Underweight $(<19)$ & 24 & 0.4 \\
\hline Index* & Normal (20-25) & 898 & 13.6 \\
\hline \multirow[t]{3}{*}{$\left(\mathrm{Kg} / \mathrm{m}^{2}\right)$} & Overweight (26-29) & 3890 & 59.0 \\
\hline & Obese Class I (30-35) & 1481 & 22.5 \\
\hline & Obese Class II-III (>36) & 299 & 4.5 \\
\hline Duration of T2DM & $0-5$ & 2939 & 44.4 \\
\hline \multirow[t]{8}{*}{$(\text { years })^{* *}$} & $6-10$ & 1844 & 27.8 \\
\hline & $11-15$ & 651 & 9.8 \\
\hline & $16-20$ & 267 & 4 \\
\hline & $21-25$ & 94 & 1.4 \\
\hline & $25-30$ & 41 & 0.6 \\
\hline & $31-35$ & 12 & 0.2 \\
\hline & $35-40$ & 6 & 0.1 \\
\hline & $41+$ & 4 & 0.1 \\
\hline \multirow[t]{3}{*}{ HbA1c } & Controlled $(<7 \%-53 \mathrm{mmol} / \mathrm{mol})$ & 2714 & 41 \\
\hline & Moderately Uncontrolled ( $\geq 7-7.99 \%-53-64 \mathrm{mmol} / \mathrm{mol})$ & 2957 & 44.7 \\
\hline & Uncontrolled ( $\geq 8 \%-64 \mathrm{mmol} / \mathrm{mol})$ & 943 & 14.3 \\
\hline
\end{tabular}

*According to NICE Guideline 432006 **The remaining $11.6 \%$ is of unknown disease duration.

Table 2. Hypoglycemia incidence

\begin{tabular}{llcc}
\hline & \multicolumn{2}{c}{ Frequency } & Percentage \\
\hline Hypoglycemia & Hypoglycemia & 2,779 & 41.9 \\
& $\begin{array}{l}\text { Laboratory-confirmed } \\
\text { Hypoglycemia }\end{array}$ & 1,354 & 20.4 \\
& Severe Hypoglycemia & 767 & 11.6 \\
\hline
\end{tabular}

controlled and uncontrolled. More specifically, the

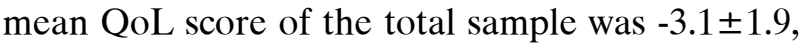
the mean score of non-hypoglycemic patients was $-3.05 \pm 2$ and the scoring of the hypoglycemic group was $-3.26 \pm 1.8,(\mathrm{p} \leq 0.001)$. This signifies that type 2
Table 3. Confirmed hypoglycemic events in controlled \& uncontrolled patients

\begin{tabular}{|c|c|c|c|c|}
\hline \multirow[t]{3}{*}{ HbA1c } & \multicolumn{4}{|c|}{ Hypoglycemia } \\
\hline & \multicolumn{2}{|c|}{ NO } & \multicolumn{2}{|c|}{ YES } \\
\hline & $\%$ & $\mathbf{N}$ & $\%$ & $\mathbf{N}$ \\
\hline Controlled & $82.8 \%$ & 2237 & $17.2 \%$ & 466 \\
\hline Uncontrolled & $77.2 \%$ & 3000 & $22.8 \%$ & 887 \\
\hline
\end{tabular}

diabetes has a greater impact on QoL when coexisting with hypoglycemia. In addition, non-hypoglycemic patients stated that their QoL would be a little better if they did not have diabetes $(-1.58 \pm 0.85)$, in contrast 


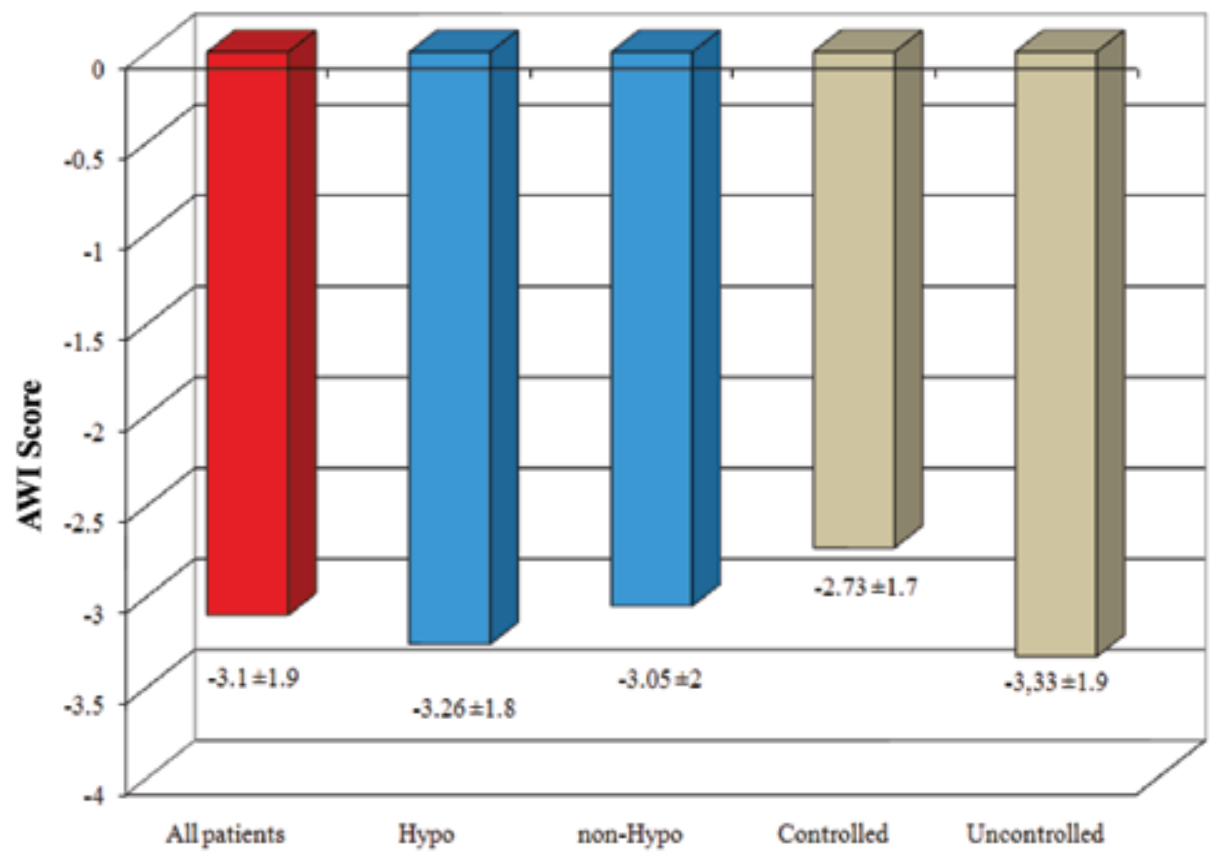

Figure 1. Average weighted QoL impact score of the study sample and subgroups. to the hypoglycemic patients who stated that it would be much better $(-1.89 \pm 0.76)$. Similar results are presented for the group of controlled patients, who scored $-2.73 \pm 1.7$ and $-3.33 \pm 1.9$ for uncontrolled, with statistical significant difference $(\mathrm{p} \leq 0.001)$.

Taking into consideration the dimensions leisure activities, working life, travel, holiday and physical activities of the ADDQoL-19, it was reflected that hypoglycemia significantly affects them. Specifically, the most influenced dimension was the "holiday". However, hypoglycemic and uncontrolled individuals' QoL were more affected compared to the nonhypoglycemic and controlled.

Regarding the dimensions family life, social life, personal life and sex life, hypoglycemia patients confronted more difficulties than non-hypoglycemia with the lowest score being noted in the dimension of sex life. Similar results were found in the second group.

Hypoglycemic patients seemed to have been more negatively influenced than the non-hypoglycemic group in the dimensions of self-confidence, motivation and feelings about the future, though not in those of physical appearance and reaction from others. The same evidence was provided among the uncontrolled individuals with the exception of motivation.
The last five dimensions of the ADDQoL questionnaire (financial situation, living conditions, reliance on others, freedom to eat, freedom to drink) showed a negative effect on patients' quality of life. Hypoglycemic patients had difficulties in living conditions, freedom to eat and, especially, in reliance on others. Moreover, the dimension with the lowest mean value in both hypoglycemic and uncontrolled samples was the freedom to eat parameter, which is related to the individuals' perception about their eating ability.

Statistically significant differences were found in 10 out of the 19 ADDQoL dimensions regarding both groups ( $\mathrm{p} \leq 0.05)$ and specifically in: a) leisure activities, b) working life, c) travel, d) holiday, e) physical activities, f) family life, g) social life, h) physical appearance, i) living conditions and j) reliance on others.

Logistic regression was performed between hypoglycemic and non-hypoglycemic patients and the ADDQoL-19 dimensions. The dependent variable was set in the existence of hypoglycemia or not (hypoglycemia $=1$, non-hypoglycemia $=0$ ), while the 19 dimensions of the instrument were used as independent variables. The factor of multiple determination was $\mathrm{R}^{2}=11.1 \%$. The observed values agreed in $83.7 \%$ of the total observations. Table 4 presents the regression model with the odds ratios for all quality of life parameters 
of the study sample for both hypoglycemia and control levels. More specifically, if diabetic patients scored 1 unit less in working life, then the relative probability of the occurrence of hypoglycemia will decrease by 0.0111 , for physical activities by 0.0136 , reliance on others by 0.0122 and 'if I didn't have diabetes my quality of life would be better' by 0.0222 . In the event that diabetic patients scored 1 unit extra, then the relative probability of the occurrence of hypoglycemia in diabetic patients will increase in the parameters of personal life by 0.0116 , reaction from others 0.0124 , financial situation 0.0110 , freedom to drink 0.0057 and, in general, quality of life by 0.0536 . A similar logistic regression model was also carried out for controlled and uncontrolled patients. Specifically, the coefficient determination was $\mathrm{R}^{2}=0.0109$. If diabetic patients answered 1 unit less in the quality of life questionnaire, then the relative probability of being controlled will decrease in all dimensions, apart from holiday, and in general 'my present quality of life is...', which have a positive association.

\section{DISCUSSION}

Hypoglycemia is an important element affecting the QoL of T2DM patients. Based on the results of the present analysis, it is observed that hypoglycemic events and severe hypoglycemic events are higher than those presented in the international literature. ${ }^{26,27}$ During the first decade of the UK Prospective Diabetes Study (UKPDS), mean proportion of patients with any hypoglycemic episode ranged from $1.2 \%$ with diet alone to $11-17 \%$, with a sulphonylurea reaching $36.5 \%$ with insulin. During the same period, major hypoglycemic episodes ranged between $0.4 \%-0.6 \%$ for sulphonylureas and were $2.3 \%$ for insulin. ${ }^{26}$ Similarly, a population-based study of health services published in 2003 reported that $7.3 \%$ of patients with T2DM

Table 4. Logistic regression analysis for hypoglycemia and control levels

\begin{tabular}{lccccc}
\hline Subscale ADDQOL-19 & \multirow{2}{*}{ B } & ODDS RATIO & \multicolumn{2}{c}{ 95\% Confidence Interval } & \multirow{2}{*}{ P value } \\
\cline { 5 - 6 } Hypoglycemia & & & Lower & Upper & \\
\hline Working Life & -0.117 & 0.889 & 0.835 & 0.947 & 0.001 \\
Physical Activities & -0.082 & 0.922 & 0.864 & 0.983 & 0.012 \\
Personal Life & 0.110 & 1.116 & 1.047 & 1.190 & 0.001 \\
Reaction from Others & 0.117 & 1.124 & 1.053 & 1.200 & 0.001 \\
Financial Situation & 0.105 & 1.110 & 1.045 & 1.180 & 0.001 \\
Reliance on Others & -0.130 & 0.878 & 0.831 & 0.928 & 0.001 \\
Freedom to Drink & 0.056 & 1.057 & 1.001 & 1.117 & 0.045 \\
In general, my present quality of life is... & 0.429 & 1.536 & 1.369 & 1.723 & 0.001 \\
if I did not have diabetes, my quality of life would be... & -0.251 & 0.778 & 0.660 & 0.918 & 0.003 \\
Constant & -2.299 & 0.100 & 0.895 & 1.017 & 0.001 \\
\hline Subscale ADDQOL-19 & $\mathbf{B}$ & $\mathbf{O D D S ~ R A T I O}$ & $\mathbf{9 5 \%}$ Confidence Interval & P value \\
Control Levels & & & Lower & Upper & \\
\hline Leisure Activities & -0.580 & 0.942 & 0.896 & 0.989 & 0.017 \\
Holiday & 0.052 & 1.054 & 1.007 & 1.103 & 0.025 \\
Family Life & -0.050 & 0.951 & 0.907 & 0.998 & 0.040 \\
Sex Life & -0.042 & 0.959 & 0.920 & 0.999 & 0.046 \\
Reaction from Others & -0.052 & 0.949 & 0.904 & 0.996 & 0.034 \\
Reliance on Others & -0.069 & 0.933 & 0.894 & 0.975 & 0.002 \\
Freedom to Drink & -0.066 & 0.937 & 0.899 & 0.976 & 0.002 \\
In general, my present quality of life is... & 0.324 & 1.382 & 1.260 & 1.516 & 0.001 \\
Constant & -1.492 & 0.225 & 0.896 & 0.989 & 0.001 \\
\hline
\end{tabular}


treated with insulin and $0.8 \%$ of patients treated via oral hypoglycemic therapy suffered at least one severe hypoglycemic event. ${ }^{27}$ On the other hand, in the UK Hypoglycemia Group Study, 7\% of severe hypoglycemic events have been reported in the population, which is much higher than previous reported studies. ${ }^{3}$ In the current study, the percentages of laboratoryconfirmed hypoglycemia was $20.4 \%$, which is quite similar to those of the UKPDS study. However, in the case of severe hypoglycemia, a great difference is recorded, with $11.6 \%$ having experienced at least one severe event, which is similar to insulin-treated patients. The high number of hypoglycemic events might be attributed to the relatively higher percentage of controlled and moderately uncontrolled patients, leading to higher frequency of hypoglycemic events. Alternatively, the difference might be due to the under-reporting of severe hypoglycemic events in previous studies. In the current study, the number of controlled patients was $41 \%$, which is in accordance with the control levels reported in the international literature (35-59\%). ${ }^{28-30}$ Furthermore, the hypoglycemia in patients with T2DM is clinically assessed on the basis of administered treatment profiles. ${ }^{30-33}$

The findings concerning patients' QoL of the current study have both similarities and differences from the international literature. The major comparable result of the current study and the international literature reveals that hypoglycemic and uncontrolled patients have lower QoL than their controlled counterparts and patients without hypoglycemia. ${ }^{4,7,32-37}$ However, lack of consistency is noticed in the international literature in terms of hypoglycemic event classification and QoL questionnaires used in order to capture the different utility levels. . $^{4,910,12,14}$ The questionnaire used to capture QoL in the current study was the ADDQOL-19 and only Costa FA et $\mathrm{al}^{138}$ have used this hypoglycemia-specific QoL questionnaire. In most of the international literature the generic instrument EQ-5D was used. ${ }^{4,10,17,30,31,39-40}$ Additionally, certain methodological differences may be observed between the current study and the international literature. In the current study, mainly patients with T2DM on oral antidiabetic drugs (OADs) were recruited, which might be considered as a limitation of the study. The only studies reporting QoL data from OADs were those of Marrett et al and Solli et al., ${ }^{417}$ Nevertheless, it is not clear whether hypoglycemia events emerging from OADs or insulin are of the same severity and what their impact is on patient's quality of life.

As far as the instrument's dimensions are concerned, both hypoglycemia and high level of HbA1c negatively affect the self-perceived QoL and, more specifically, the dimensions of holidays, sex life, selfconfidence, travel, motivation, feelings about the future, living conditions, freedom to eat and reliance on others. ${ }^{11,35}$ Based on the current study, hypoglycemia as well as high levels of $\mathrm{HbA1c}$ were negatively correlated with the dimensions of the ADDQoL-19 instrument.

There is a paucity of studies in the literature on the measurement of the QoL of diabetic patients with the ADDQoL-19 instrument. The results of the present study are likely to contribute to filling this gap in our knowledge about health-related QoL measurement for diabetic patients and the need for effective health strategies and social policies in the field of diabetic treatment.

\section{CONCLUSION}

The current results highlight the fact that patients experiencing hypoglycemia and uncontrolled patients confront significant restrictions in many dimensions of their living conditions. In addition, the existence of hypoglycemia and high levels of $\mathrm{HbA} 1 \mathrm{c}$ has a negative impact on important aspects of QoL and well-being of patients. Effective health policy interventions need to focus on specific treatments and strategies aiming at improving the overall well-being of diabetic patients. In diabetes treatment, the ultimate goal should be good glycemic control without debilitating hypoglycemic episodes which compromise patients' QoL.

\section{REFERENCES}

1. International Diabetes Federation, IDF Diabetes Atlas, 2012 Available from http://www.idf.org/diabetesatlas /5e/Update 2012. Accessed 20 December 2012

2. Panagiotakos DB, Pitsavos C, Chrysohoou C, et al, 2005 The epidemiology of Type 2 diabetes mellitus in Greek adults: the ATTICA study. Diab Med 22: 1581-1588.

3. UK Hypoglycemia Study Group, 2007 Risk of hypo- 
glycemia in types 1 and 2 diabetes: effects of treatment modalities and their duration. Diabetologia 50: 1140-1147.

4. Solli O, Stavem K, Kristiansen IS, 2010 Health-related QoL in diabetes: The associations of complications with EQ-5D scores. Health and QoL Outcomes 8: 18.

5. Bradley C, 2001 FIT: A paradigm shift that recognizes the importance of dietary freedom and QoL in diabetes management. Health Psychology 10: 74-75.

6. BotijaYagüe MP, LizanTudela L, Gosalbes Soler V, et al, 2007 How does intensive therapy to control cardiovascular risk factors affect health-related QoL in diabetic patients? Atención Primera 39: 227-234.

7. Tabaei BP, Shill Novak J, Brandle M, et al, 2004 Glycemia and the quality of well-being in patients with diabetes. QoL Research 13: 24-28.

8. Petterson T, Lee P, Hollis S, et al, 1998 Well-being and treatment satisfaction in older people with diabetes. Diabet Care 21: 930-935.

9. Testa M, 2000 Quality-of-Life assessment in diabetes research: interpreting the magnitude and meaning of treatment effects. Diabetes Spectrum 13: 29.

10. Currie CJ, Morgan CL, Poole CD, et al, 2006 Multivariate models of health-related utility and the fear of hypoglycemia in people with diabetes. Curr Med Res Opin 22: 1523-1534.

11. Bradley C, Speight J, 2000 Patient perceptions of diabetes and diabetes therapy: assessing quality of life. Diabetes Metab Res Rev 2002;18:S64-9 Rubin R. Diabetes and quality of life. Diabetes Spectrum 13: 21.

12. Luscomble F, 2000 Health related QoL measurement in type 2 diabetes. Value in Health 3: 15-28.

13. Franciosi M, Pellegrini F, De Berardis G, et al, 2001 The impact of blood glucose self - monitoring on metabolic control and QoL in type 2 diabetic patients. Diabetes Care 24: 1870-1877.

14. Grandy S, Fox K, 2008 EQ-5D visual analogue scale and utility index values in individuals with diabetes and at risk for diabetes: findings from the study to help improve early evaluation and management of risk factors leading to diabetes (SHIELD). Health and QoL Outcomes 6:18.

15. Holmes J, McGill S, Kind P, et al, 2003 Health-related QoL in type 2 diabetes (TARDIS-2). Value Health 3: Suppl 1: 47-51.

16. Rubin RR, Peyrot M, 1999 QoL and diabetes. Diabetes Metab Res 15: 205-218.

17. Marrett E, Stargardt T, Mavros P, et al, 2009 Patientreported outcomes in a survey of patients treated with oral antihypertensive medications: associations with hypoglycaemia and weight gain. Diabetes Obes Metab 11: 1138-1144.

18. Wexler D, Grant R, Wittenberg E, et al, 2006 Correlates of health-related QoL in type 2 diabetes. Diabetologia 49: 1489-1497.
19. Palitzsch K, Volmer T, Abrams C, et al, 2000 The impact of diabetes risk factors on the QoL of patients with type 2 diabetes. Diabetes 49: 390.

20. Massi-Benedetti M, 2002 The cost of diabetes type II in Europe. The CODE-2 Study. Diabetologia 45: S1-S4.

21. Hill-Briggs F, Gary TL, Hill MN, et al, 2002 Healthrelated QoL in urban African Americans with Type 2 Diabetes. J Gen Intern Med 17: 412-419.

22. American Diabetes Association, 2008 Economic costs of diabetes in the US in 2007. Diabetes Care 31: 1-20.

23. Bradley C, Todd C, Gorton T, et al, 1999 The development of an individualized questionnaire measure of perceived impact of diabetes on quality of life: the ADDQoL. QoL Research 8: 79-91.

24. Fayers P, Machin D, 2000 Quality of life, assessment, analysis and interpretation. J Willey's, England.

25. Cohen J, 1998 Statistical power analysis for the behavioral science. Lawrence Erlbaum, Hillsdale, New Jersey.

26. UK Prospective Diabetes Study (UKPDS) Group, 1998 Intensive blood-glucose control with sulphonylureas or insulin compared with conventional treatment and risk of complications in patients with type 2 diabetes (UKPDS 33). UK Prospective Diabetes Study (UKPDS) Group. Lancet 352: 837-853.

27. Leese GP, Wang J, Broomhall J, et al, 2003 Frequency of severe hypoglycemia requiring emergency treatment in type 1 and type 2 diabetes. Diabetes Care 26: 1176-1180.

28. Koro CE, Bowlin SJ, Bourgeois N, Fedder DO, 2004 Glycemic control from 1988 to 2000 among U.S. adults diagnosed with type 2 diabetes: a preliminary report. Diabetes Care 27: 17-20.

29. Ong KL, Cheung BM, Wong LY, Wat NM, Tan KC, Lam KS, 2008 Prevalence, treatment, and control of diagnosed diabetes in the U.S. National Health and Nutrition Examination Survey 1999-2004. Ann Epidemiol. 18: 222-229.

30. Zammitt NN, Frier BM, 2005 Hypoglycemia in type 2 diabetes: pathophysiology, frequency, and effects of different treatment modalities. Diabetes Care 28: 2948-2961.

31. Miller CD, Phillips LS, Ziemer DC, et al, 2001 Hypoglycemia in patients with type 2 diabetes mellitus. Arch Intern Med 161: 1653-1659.

32. Philip E, Cryer A, 2008 The barrier of hypoglycemia in diabetes. Diabetes 57: 3169-3176.

33. Alvarez-Guisasola F, Yin DD, Nocea G, et al, 2010 Association of hypoglycemic symptoms with patients' rating of their health-related QoL state: a cross sectional study. Health and QoL Outcomes 8: 86.

34. Lundkvist J, Berne C, Bolinder B, et al, 2005 The economic and QoL impact of hypoglycemia. Eur J Health Econ 6: 197-202.

35. Singh H, Bradley C, 2006 QoL in diabetes, Int J Diab Dev Ctries 1: 1.

36. Goddijn PP, Bilo HJ, Feskens EJ, et al, 1999 Longitudinal 
study on glycaemic control and QoL in patients with type 2 diabetes mellitus referred for intensified control. Diabet Med 16: 23-30.

37. Ghanbar A, Yekta ZP, Roushan ZA, et al, 2005 Assessment of factors affecting QoL in diabetic patients in Iran. Public Health Nurs 22: 311-322.

38. Costa FA, Guerreiro JP, Duggan C, 2006 An Audit of Diabetes Dependent QoL (ADDQoL) for Portugal: exploring validity and reliability. Pharmacy Practice
4: 123-128.

39. Levy AR, Christensen TL, Johnson JA, 2008 Utility values for symptomatic non severe hypoglycemia elicited from persons with and without diabetes in Canada and the United Kingdom. Health Qual Life Outcomes 6: 73.

40. Matza LS, Boyle KS, Yurgin N, et al, 2007 Utilities and disutilities for type 2 diabetes treatment-related attributes. Qual Life Res 16: 1251-1265. 